\title{
During the COVID-19 pandemic, oral health services including routine treatment may continue but must follow established international and South African protocol
}

SADJ August 2020, Vol. 75 No. 7 p351 - p352

KC Makhubele
CEO of SADA

The South African Dental Association (SADA) hereby responds to the statement released by the World Health Organization (WHO): Considerations for the provision of essential oral health services in the context of COVID-19, on $7^{\text {th }}$ August 2020

After careful consideration and in consultation with workstreams working in various protocols regarding the provision of oral health services during the COVID-19 pandemic, SADA respectfully yet strongly disagrees with the World Health Organization's (WHO) recommendation to delay "routine" dental care in certain situations due to COVID-19.

A blanket statement such as the recent one by the WHO cannot, therefore, be supported. It is without a doubt that oral health is integral to overall health and neglect and delay of certain treatments may lead to extensive damage and increased financial implications to correct.

Consequently, dentistry is essential healthcare, because of its role in evaluating, diagnosing, preventing or treating oral diseases, which can adversely affect systemic health. Each country is at a different stage of the pandemic and each country has to respond appropriately based on its assessment of its unique situation.

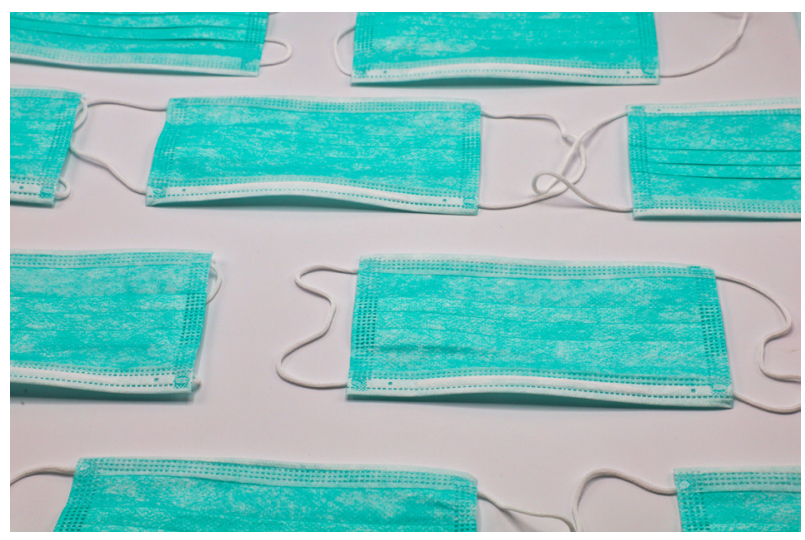

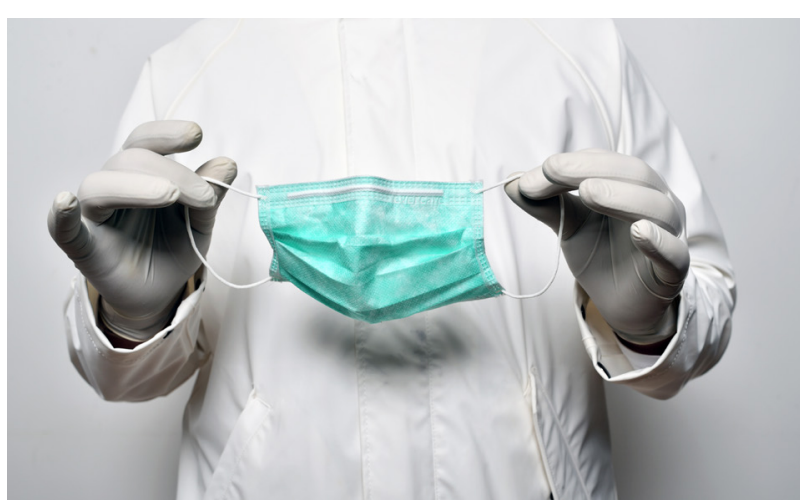

The South African Government has set out clear guidelines at various stages of alert. This is the context in which the profession operates.

Our stance is supported by the decision of the FDI World Dental Federation, which SADA belongs to. The FDI is made by approximately 200 country (National) dental associations in about 130 countries internationally. Following the general provisions of the WHO regarding oral health services, SADA published a well researched and widely supported protocols - The SADA Dental Clinical Protocol, available at www.sada.co.za, to ensure that patients, staff and healthcare workers are adequately protected.

The SADA Dental Clinical Protocol to which all dentists should adhere rests on the following four pillars:

1. Proper ventilation of the practice.

2. The use of high-volume evacuation (HVE) to evacuate aerosols.

3. The use of rubber dams (a protective film to isolate the oral cavity) whenever possible in combination with HVE.

4. The correct use of appropriate PPE during each procedure. 
SADA, therefore, support the FDI statement that "... oral healthcare is essential to maintaining good overall health and routine care is necessary for the early detection, prevention, and control of oral diseases.

FDI advocates that every opportunity should be utilized to reinforce oral health promotion and prevention messages to help reduce the need for avoidable dental visits. But, at the same time, governments must ensure continued and equitable access to essential oral healthcare services, as well as the availability of appropriate personal protective equipment (PPE), to avoid an even bigger burden on health systems in the future."

In line with the general healthcare preparations made to cope with the first wave of expected COVID-19 cases, SADA, with unprecedented support from its dentistry community, formalised a clinical workstream to establish a protocol for how oral healthcare workers (OHCWs) could work during the pandemic.

We realised that we would need to be active during times of high viral load, as the threat of infection was very low during our actual lockdown.

The dental environment is naturally high-risk due to the unavoidable contact between patients and OHCWs, which increases the possibility of transmitting HIV, resistant $\mathrm{TB}$, etc.

Stringent standard of care in the sterilisation and disinfection protocols is thus standard practice. However, the possibility of an airborne vector needed to be incorporated into our standard operating protocol. We acknowledge that there is still much to be learned about the coronavirus, therefore we adopted the route of the worst-case scenario.

We accepted the possibility of airborne spread very early (March 2020) and considered the scope of the virus in preparing a document containing clinical standards and procedures under which the OHCW's could function safely and the health of $\mathrm{OHC}$ staff, patients, and the population at large would be safeguarded.

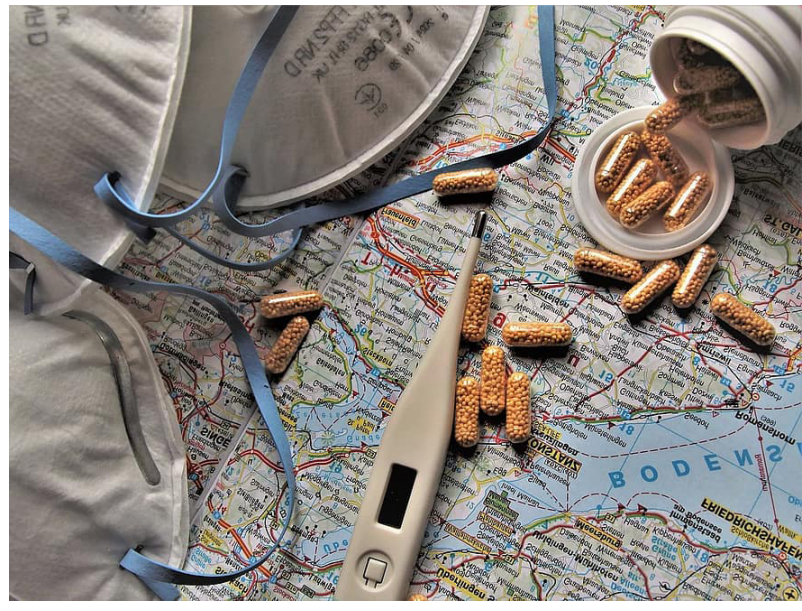

This document included opinions from South African dentists, the academic dental deans of all South African universities, specialists, experts in the field and the dental legal fraternity.

SADA recognizes the need for our patients not to be denied any treatment that could be detrimental to their oral or general health. In light of this principle during lockdown Level 3 and below, most dental procedures are permissible, with strict adherence given to proper protocol.

Routine dental visits are therefore encouraged where a dental practice strictly adheres to the SADA and other relevant protocols. The public at large should not unnecessarily delay treatment if a need arises.

Where members of the public are concerned, they are welcome to source information from our website and or contact the SADA offices.

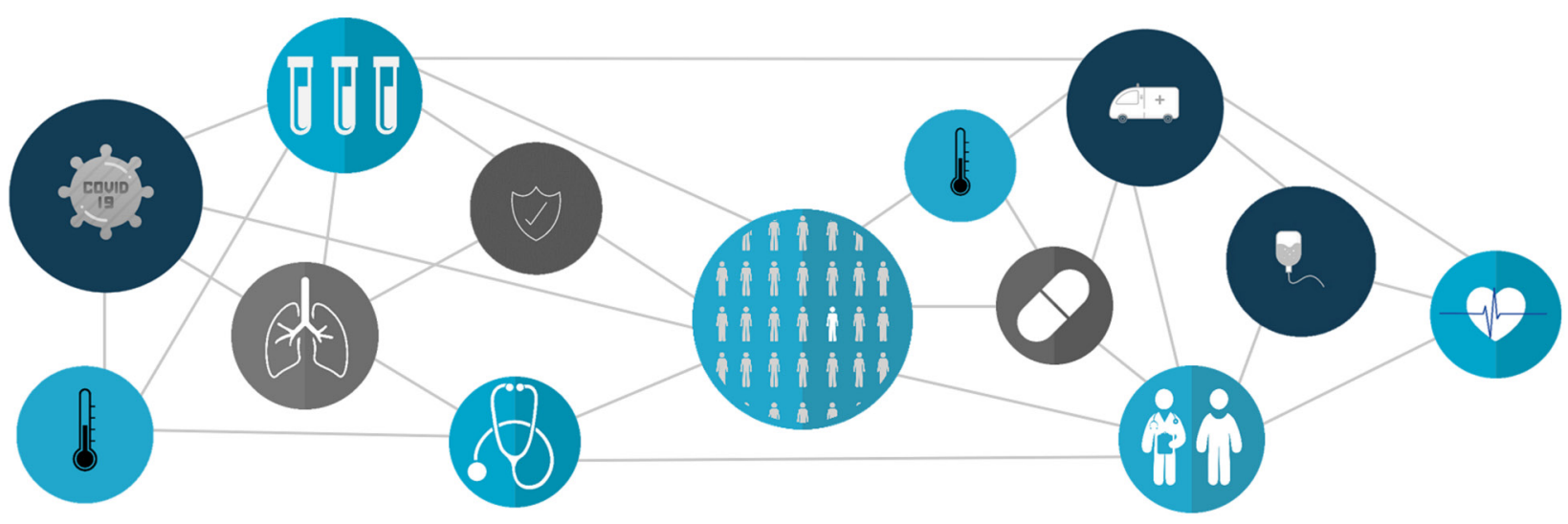

